\title{
Impairment of Sharp-Wave Ripples in a Murine Model of Dravet Syndrome
}

\author{
Christine S. Cheah, ${ }^{1}$ Brian N. Lundstrom, ${ }^{3}$ William A. Catterall, ${ }^{2}$ and John C. Oakley ${ }^{1,2}$ \\ Departments of ${ }^{1}$ Neurology, ${ }^{2}$ Pharmacology, University of Washington, Seattle, Washington 98195 , and ${ }^{3}$ Department of Neurology, Mayo Clinic, Rochester, \\ Minnesota 55905
}

Dravet syndrome (DS) is a severe early-onset epilepsy associated with heterozygous loss-of-function mutations in SCN1A. Animal models of DS with global Scnla haploinsufficiency recapitulate the DS phenotype, including seizures, premature death, and impaired spatial memory performance. Spatial memory requires hippocampal sharp-wave ripples (SPW-Rs), which consist of high-frequency field potential oscillations (ripples, $100-260 \mathrm{~Hz}$ ) superimposed on a slower SPW. Published in vitro electrophysiologic recordings in DS mice demonstrate reduced firing of GABAergic inhibitory neurons, which are essential for the formation of SPW-R complexes. Here, in vivo electrophysiologic recordings of hippocampal local field potential in both male and female mice demonstrate that Scn1a haploinsufficiency slows intrinsic ripple frequency and reduces the rate of SPW-R occurrence. In DS mice, peak ripple-band power is shifted to lower frequencies, average intertrough intervals of individually detected ripples are slower, and the rate of SPW-R generation is reduced, while SPW amplitude remains unaffected. These alterations in SPW-R properties, in combination with published reductions in interneuron function in DS, suggest a direct link between reduced inhibitory neuron excitability and impaired SPW-R function. A simple interconnected, conductance-based in silico interneuron network model was used to determine whether reduced sodium conductance is sufficient to slow ripple frequency, and stimulation with a modeled SPW demonstrates that reduced sodium conductance alone is sufficient to slow oscillatory frequencies. These findings forge a potential mechanistic link between impaired SPW-R generation and Scn la mutation in DS mice, expanding the set of disorders in which SPW-R dysfunction contributes to impaired memory.

Key words: channelopathy; Dravet syndrome; epilepsy comorbidity; learning and memory; Scn1a; sharp-wave ripple

Significance Statement

Disruption of sharp-wave ripples, a characteristic hippocampal rhythm coordinated by the precise timing of GABAergic interneurons, impairs spatial learning and memory. Prior in vitro patch-clamp recordings in brain slices from genetic mouse models of Dravet syndrome (DS) reveal reduced sodium current and excitability in GABAergic interneurons but not excitatory cells, suggesting a causal role for impaired interneuron activity in seizures and cognitive impairment. Here, heterozygous Scn1a mutation in DS mice reduces hippocampal sharp-wave ripple occurrence and slows internal ripple frequency in vivo and a simple in silico model demonstrates reduction in interneuron function alone is sufficient to slow model oscillations. Together, these findings provide a plausible pathophysiologic mechanism for Scnla gene mutation to impair spatial memory.

\section{Introduction}

Dravet syndrome (DS) is a severe pharmacoresistant infantileonset epileptic encephalopathy associated with mutations in $S C N 1 A$, the gene encoding the $\alpha$-subunit of the brain type I voltage-gated sodium channel $\mathrm{Na}_{\mathrm{v}} 1.1$. DS is characterized by fre-

\footnotetext{
Received April 16, 2019; revised Sept. 3, 2019; accepted Sept. 4, 2019.

Author contributions: C.S.C., B.N.L., W.A.C., and J.C.O. designed research; C.S.C., B.N.L., and J.C.O. performed research; C.S.C., B.N.L., and J.C.O. analyzed data; C.S.C., B.N.L., and J.C.O. wrote the paper.

The research reported in this publication was supported by the National Institutes of Neurological Disorders and Stroke of the National Institutes of Health under Grants K08-NS-071193 and R01-NS-094186 to J.C.0., and R01-NS25704 to W.A.C.

The authors declare no competing financial interests.

Correspondence should be addressed to John C. 0akley at jcoakley@uw.edu.

https://doi.org/10.1523/JNEUROSCI.0890-19.2019

Copyright $@ 2019$ the authors
}

quent, prolonged seizures and comorbidities including autistic features, intellectual disability, and memory impairment (Chieffo et al., 2011b; Dravet, 2011). Classically, cognitive impairments in epileptic encephalopathies, such as DS, are attributed to poor seizure control. Alternatively, an underlying molecular-genetic defect could directly impact network function resulting in both intellectual disability and seizures independently. A prospective clinical study suggests that the severity of the SCN1A mutation directly modulates cognitive impairment (Nabbout et al., 2013). If correct, aggressive medical therapy for seizure control may not significantly improve long-term cognitive outcomes.

The globally haploinsufficient DS mouse is a faithful genetic and phenotypic mimic of the human disease, making it an ideal model to study the impact of Scn1a mutation on neural circuit 
function. A high proportion of sodium current in GABAergic interneurons (INs) is provided by $\mathrm{Na}_{\mathrm{v}} 1.1$, and prior work in both DS mice and conditional Scn1a mutants has shown reduced sodium current and decreased excitability in all IN subtypes (Yu et al., 2006; Kalume et al., 2007; Ogiwara et al., 2007; Han et al., 2012b; Tai et al., 2014). In contrast, excitatory pyramidal cells express a high level of other neuronal sodium channels (Vacher et al., 2008), which is consistent with the unchanged excitatory neuron function observed in DS mice (Yu et al., 2006; Tai et al., 2014) and the finding that the mutation of Scnla in excitatory neurons does not cause DS phenotypes (Ogiwara et al., 2013).

Coordinated patterns of activity in neural circuits are detectable in local field potential (LFP) recordings. Distinct rhythms are associated with specific behaviors such as sleep, exploration, learning, and memory, but disrupted brain rhythms can emerge in epilepsy and other neuropsychiatric diseases (Traub, 2003; Hutchison et al., 2004; Jeong, 2004; Timofeev et al., 2004; van der Stelt et al., 2004). For example, pathologic high-frequency oscillations (pHFOs; $250-600 \mathrm{~Hz}$ ) have been detected in regions of epileptogenesis in both animals and humans (Bragin et al., 2002; Jefferys et al., 2012) and reduced inhibition in hippocampal brain slices in vitro and in vivo results in pHFO (Bragin et al., 1999; Gulyás and Freund, 2015). Scn1a mutation-related reduction in sodium current and impaired firing of GABAergic INs demonstrated in DS mice are expected to significantly impact network functions dependent on those cell classes (Hu et al., 2014).

Hippocampal sharp-wave ripple (SPW-R) complexes are well characterized, high-frequency $(140-220 \mathrm{~Hz})$ brain rhythms that are critical for short-term and long-term spatial learning and memory (Bartos et al., 2007; Girardeau and Zugaro, 2011; Gulyás and Freund, 2015), both of which are impaired in patients with DS and DS mice (Chieffo et al., 2011a; Han et al., 2012a; Ito et al., 2013). SPW-R complexes recorded in CA1 have the following two components: the SPW, a slow extracellular negative potential with maximal amplitude in CA1 stratum radiatum; and ripples, fast oscillations $(140-220 \mathrm{~Hz})$ superimposed on the SPW (Buzsáki, 2015). Both the initiation of the SPW and the coordination of activity underlying ripples are dependent on intact IN function to pace the excitation of local pyramidal cells, which are responsible for generating the LFP signal recognized as an SPW-R (Schlingloff et al., 2014).

This study finds that global $\mathrm{Na}_{\mathrm{v}} 1.1$ haploinsufficiency in DS mice reduces the rate of individual SPW-R complex occurrence and slows internal ripple frequency oscillation. A simple in silico interconnected interneuron model with reduced sodium conductance demonstrates a similar slowing of oscillatory frequency. Building on previous findings of reduced interneuron excitability in DS mice (Yu et al., 2006; Ogiwara et al., 2007), the dependence of SPW-R on GABAergic interneuron activity (Schlingloff et al., 2014; Stark et al., 2014), and the necessity of SPW-R for spatial memory (Girardeau et al., 2009), the findings in this study provide a mechanistic link among Scnla gene mutation, interneuronal hypoexcitability, and cognitive and behavioral impairment.

\section{Materials and Methods}

Mouse strains and general experimental design. Scn1a mutant mice were generated by targeted deletion of the last exon encoding domain IV from the S3-S6 segments and the entire C-terminal tail of $\mathrm{Na}_{\mathrm{V}} 1.1$ channels as described previously (Yu et al., 2006). Mice in this study were generated by crossing heterozygous mutant males of $\mathrm{C} 57 \mathrm{BL} / 6 \mathrm{~J}$ background with wild-type (WT) C57BL/6J females to generate heterozygous mutant mice, the Dravet disease model, and wild-type littermates. Genotype was confirmed using a four-oligonucleotide multiplex PCR of genomic DNA samples isolated from mouse tails (Yu et al., 2006). To minimize the number of mice, a mix of male and female mice were used. All experiments were performed according to guidelines established in the National Institutes of Health National Research Council, 2011 and were approved by the University of Washington Institutional Animal Care and Use Committee.

Surgery. Adult mice (WT, $n=5$; DS, $n=7$; postnatal day 30 or greater) used in this study were housed in standard housing cages in the University of Washington Animal Facility under specific pathogen-free conditions with constant temperature and a $12 \mathrm{~h}$ light/dark cycle. All animals were naive to other experimental protocols before surgery. PFA-coated stainless steel wire LFP electrodes (diameter: bare, $76.2 \mu \mathrm{m}$; coated, 139.7 $\mu \mathrm{m}$; A-M Systems) were implanted aseptically using stereotactic guidance (David Kopf Instruments) under ketamine/xylazine (130/8.8 mg/ $\mathrm{kg}$ ) anesthesia (adapted from Kalume et al., 2013). Electrodes were inserted through small burr holes and targeted to bilateral dorsal hippocampus at the junction between the pyramidal and radiatum layers where high-frequency ripple and slower sharp-wave activities are both present (Bregma, $-1.75 \mathrm{~mm}$; Depth, $-1.1 \mathrm{~mm}$; Lateral, $-1.25 \mathrm{~mm}$ ). Electrode locations were confirmed by characteristic electrophysiologic patterns (Ylinen et al., 1995) and histology (cresyl violet Nissl stain, 50 $\mu \mathrm{m}$ slices, $4 \%$ PFA transcardial perfusion). Stainless steel fine-wire electrodes placed in bilateral neck extensor muscles allowed the recording of electromyogenic (EMG) activity. Screw electrodes served as ground and reference. Electrodes were fixed in place using dental cement, and the skin was closed with sutures. Mice were allowed to recover for $>48 \mathrm{~h}$ before recordings. Following surgery, animals were singly housed in standard home cages until completion of the LFP recordings.

Local field potential recordings. On the day of recordings, mice were placed in a Plexiglas recording chamber during the light phase of the day and a rectal thermocouple was inserted allowing monitoring of body core temperature (Physitemp Instruments). A digital recording system (Tucker-Davis Technologies) was used to record hippocampal LFP (6 $\mathrm{kHz})$, neck extensor EMG (6 kHz), and body temperature $(10 \mathrm{~Hz})$. Offline, LFP and EMG records were antialias filtered and downsampled to $2048 \mathrm{~Hz}$ for subsequent analysis.

Frequency analysis. Estimates of LFP recording spectral density were generated using the periodogram approach by subdividing the recorded signal into multiple overlapping segments, computing the windowed (Hanning) normalized Fourier transform of each segment, and subsequently averaging the results over all segments (Igor, WaveMetrics; Harris, 1978). Instantaneous frequency-specific power was determined by Hilbert transform of bandpass-filtered recording. Bandpass filtering was performed off-line with finite impulse response-based digital filtering using 101 coefficients. High- and low-pass frequencies used include delta $(1.7-3 \mathrm{~Hz})$, theta $(5-12 \mathrm{~Hz})$, sharp wave $(10-20 \mathrm{~Hz})$, gamma $(30-80$ $\mathrm{Hz})$, ripple $(100-260 \mathrm{~Hz})$, and EMG $(200-700 \mathrm{~Hz})$. Spectral density analysis indicates minimal power in higher $200-600 \mathrm{~Hz}$ bands where pathologic high-frequency oscillations are observed in hippocampal LFP recordings from rodent models of temporal lobe epilepsy. Ripple-band frequencies were selected to best describe the range in which increased power is seen in the entire cohort of animals.

Behavioral state analysis. State classification was based on neck extensor muscle activity and automated identification of characteristic hippocampal rhythms based on well characterized activities associated with each state. Specifically, theta and gamma activity with high muscle activity is present during periods of exploration, high-amplitude irregular hippocampal activity with low muscle activity characterizes sleep, and high-amplitude irregular activity with moderate muscle activity is observed during quiet wakefulness (rest). Example periods of sleep, rest, and movement were visually identified for normalization of power and confirmation of correct classification. The recording was subdivided into consecutive $1 \mathrm{~s}$ windows, and for each window average EMG, theta, and gamma power were determined and $z$-score was normalized by the SD of power in the identified period of sleep. The threshold between low and high power was set at 3 SDs and manually adjusted to optimize correct classification of identified examples in each state. Normalized power was used to ensure comparable thresholds across mice and genotypes. Windows with low EMG power were classified as sleep, while windows with 
high EMG power were classified as exploration if theta and gamma power were high or as rest if theta and gamma power were low. Classification was then reviewed and corrected as needed based on visual inspection.

Sharp-wave ripple identification. Individual ripple identification was performed off-line using an automated approach based on transient increases in ripple-band $(100-260 \mathrm{~Hz})$ power. To control for potential differences in ripple signal-to-noise ratio across recording sites and genotypes, instantaneous ripple power was $z$-score normalized using the average and SD of power during a visually identified example of sustained sleep. Peaks in the $z$-scored instantaneous power $>2$ SDs from the sleep mean were identified, and for each identified peak the time at which power traversed $0.5 \mathrm{SD}$ before and after was used to define the start and end of a ripple candidate region. Within this region, troughs were identified in the ripple bandpass-filtered recording. Troughs detected $<4$ or $>10 \mathrm{~ms}$ (corresponding to $100-260 \mathrm{~Hz}$ ) from the last identified trough were excluded. The detection of four or more troughs (three or more intervals) was used to define a ripple. Start and end times of each SPW-R were defined by the first and last detected trough. For each detected ripple, the average frequency and ripple-band power were determined by averaging instantaneous frequency and power over the five middle trough intervals (three intervals for ripples containing fewer than six ripple troughs). Using this method, no difference was found in the distribution of detected SPW-Rs as recorded trough amplitudes among mice, genotypes, or recording sites, ensuring that recording site variation did not systematically impact ripple signal-to-noise and bias ripple detection and subsequent analysis. Instantaneous frequency was calculated based on intertrough intervals, while instantaneous power was determined from Hilbert transform of ripple bandpass filtered LFP as described above. Average ripple frequency, binned by ripple amplitude, is slower in DS across the entire amplitude range, demonstrating that reduced ripple frequency in DS mice is not due to a difference in the recording or detection of a ripple. / the total number of detected ripples by genotype was as follows: WT, $\sim 16,700$ ripples; DS, $\sim 14,600$ ripples. For each ripple, the corresponding sharp wave was identified in the sharp-wave bandpass filtered $(10-20 \mathrm{~Hz})$ LFP recording. SPW power was defined as the maximal instantaneous power in the SPW bandpass filtered recording during each SPW-R.

Interneuronal network model. The model consists of 100 , fully interconnected, conductance-based, single compartment model neurons with membrane properties governed by standard Hodgkin-Huxley equations (Hodgkin and Huxley, 1952; Koch, 1999; Dayan and Abbott, 2001; Gerstner and Kistler, 2002) as modified by Wang and Buzsáki (1996) to better reflect firing properties of fast-spiking interneurons. Namely, to account for differences between squid neurons, upon which the Hodgkin-Huxley neuron is based, and hippocampal fast-spiking interneurons, the kinetics and maximal conductances were modestly altered. Potassium repolarization after spiking is less pronounced, and faster kinetics of the sodium and potassium channels allow for highfrequency firing. Synapses are fast and shunting (Bartos et al., 2007).

Model cell behavior is described by the following:

$$
C_{m} \frac{d V}{d T}=-I_{\mathrm{Na}}-I_{K}-I_{L}+I_{\mathrm{SPW}}+I_{\mathrm{syn}}
$$

where $C_{m}=1 \mu \mathrm{F} / \mathrm{cm}^{2}, I_{\mathrm{Na}}$ is fast sodium current, $I_{K}$ is delayed rectifier potassium current, $I_{L}$ is leak current, $I_{\mathrm{SPW}}$ is model sharp-wave current, and $I_{\text {syn }}$ is inhibitory synaptic current (all in microamperes per square centimeter).

Assuming the fast sodium activation variable $m$, the transient sodium current is described by the following:

$$
\begin{gathered}
I_{\mathrm{Na}}=g_{\mathrm{Na}} m_{\infty}^{3} h\left(V-E_{\mathrm{Na}}\right) \\
m_{\infty}=\alpha_{m} /\left(\alpha_{m}+\beta_{m}\right) \\
\alpha_{m}(V)=-0.1(V+35) /(\exp (-0.1(V+35))-1) \\
\beta_{m}(V)=4 \exp (-((V+60) / 18))
\end{gathered}
$$

$$
\begin{gathered}
\frac{d h}{d t}=\varphi\left(\alpha_{h}(1-h)-\beta_{h} h\right) \\
\alpha_{h}(V)=0.07 \exp (-(V+58) / 20) \\
\beta_{h}(V)=1 /(\exp (-0.1(V+28))+1),
\end{gathered}
$$

where $g_{\mathrm{Na}}=35 \mathrm{mS} / \mathrm{cm}^{2}, E_{\mathrm{Na}}=55 \mathrm{mV}$, and $\varphi=5$.

The delayed rectifier potassium current is described by the following:

$$
\begin{gathered}
I_{k}=g_{k} n^{4}\left(V-E_{k}\right) \\
\frac{d n}{d t}=\varphi\left(\alpha_{n}(1-n)-\beta_{n} n\right) \\
\alpha_{n}(V)=-0.01((V+34) /(\exp (-0.1(V+34))-1)) \\
\beta_{n}(V)=0.125 \exp (-(V+44) / 80),
\end{gathered}
$$

where $g_{K}=9 \mathrm{mS} / \mathrm{cm}^{2}$ and $E_{K}=-90 \mathrm{mV}$.

Leak current is described by the following:

$$
I_{L}=g_{L}\left(V-E_{L}\right),
$$

$g_{L}=0.1 \mathrm{mS} / \mathrm{cm}^{2}$ and $E_{L}=-65 \mathrm{mV}$.

$I_{\mathrm{SPW}}$ is composed of periodic (every $100 \mathrm{~ms}$ ) Gaussian-shaped [fullwidth at half-maximal (FWHM) amplitude, $20 \mathrm{~ms}$ ] positive currents with superimposed constant Gaussian-filtered $(\tau=1 \mathrm{~ms})$ white noise $\left(\mathrm{SD}=0.5 \mu \mathrm{A} / \mathrm{cm}^{2}\right)$ simulating the CA3 to CA1 excitatory input giving rise to SPW (Csicsvari et al., 2000).

Synaptic current $I_{\text {syn }}$ was modeled by the following:

$$
I_{s y n}=g_{s y n} s\left(V-E_{s y n}\right),
$$

where the maximal synaptic conductance $g_{\text {syn }}=1 \mathrm{mS} / \mathrm{cm}^{2}$ and $E_{\text {syn }}=$ -55 . The variable $s$ is the synaptic gating variable, which presents the fraction of open synaptic ion channels governed by the following:

$$
\tau_{s y n} \frac{d s}{d t}=-s,
$$

where $\tau_{\text {syn }}=2 \mathrm{~ms}$ (Bartos et al., 2007). For each presynaptic action potential, $s$ was increased by 1 (Taxidis et al., 2012). Specifically, at each time point $s=s+W v$, where the $i$ th element of vector $v$ was 1 if the $i$ th presynaptic neuron fired an action potential (i.e., its membrane voltage was greater than $-20 \mathrm{mV}$ ). Differential equations of the network models were solved with a fourth-order Runge-Kutta method and $0.05 \mathrm{~ms}$ time step. Initial conditions were chosen from a uniform random distribution with membrane potential range $(-75$ to -55$)$ and gating variable range $(0-0.2)$; however, initial conditions were generally unimportant as the model reached equilibrium before stimulus onset. To characterize the frequency and timing of model oscillations, model ripples were displayed as the bandpass-filtered mean synaptic currents. Synaptic currents were averaged from all synapses and filtered by a second-order Butterworth filter with a passband $100-300 \mathrm{~Hz}$, as implemented by MATLAB (MathWorks). Power spectra were calculated from the unfiltered average synaptic currents for each network and then smoothed by averaging adjacent bins.

Statistical analysis. Average results from each mouse were used to generate within genotype grand averages to ensure equal weighting of each animal independent of the absolute number of observations. Quantitative data are reported as the mean \pm SEM. Statistical significance of quantitative data grouped by genotype was determined by WilcoxonMann-Whitney two-sample rank test, which does not assume normally distributed data and is more robust than an independent-samples $t$ test to outlier data points ( $\alpha=0.05$, two tailed). Effect size is reported as rankbiserial correlation $(r)$ specifying the effect size from -1 (all less than) to +1 (all greater than), with 0 indicating no relationship.

Data availability. The data that support the findings of this study are available from the corresponding author, upon reasonable request. 
A
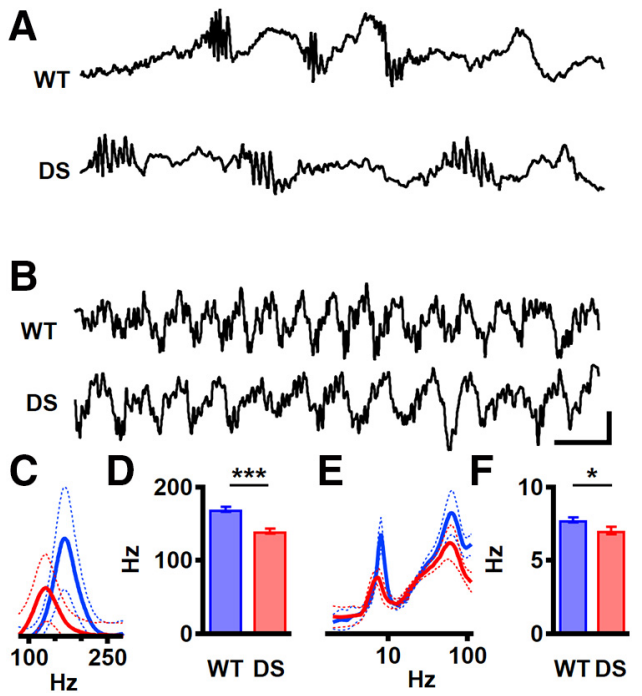

Figure 1. Gross morphology of hippocampal CA1 activities characteristic of exploration, rest, and sleep are preserved in LFP recordings, but sleep and resting state ripple and exploratory theta-band frequencies are slowed. $A, B$, Example CA1 LFP recordings during periods of sleep $(\boldsymbol{A})$ and exploration (B) in WT (top) and DS (bottom) mice demonstrate similar SPW-R morphology $(\boldsymbol{A})$ and rhythmic theta-gamma activities $(\boldsymbol{B})$. $\boldsymbol{C}$, Average frequency spectrograms by genotype (blue: WT, $n=5$; red: DS, $n=7$ ) from concatenated periods of sleep show that ripple-band frequencies are slower and have reduced power in DS mice. Genotype average \pm $S E M$. $D$, Average frequency at peak ripple power \pm SEM by genotype quantifies slower $D S$ ripple frequency (WT mice, $170 \pm 4 \mathrm{~Hz}$; DS mice, $141 \pm 4 \mathrm{~Hz} ; p=0.003, r=-1$ ). $E$, During exploration in DS mice, theta- and gamma-band power is reduced, and there is a modest reduction in frequency at peak theta power. $\boldsymbol{F}$, Genotype average $\pm S E M$. Average of frequency at peak theta power \pm SEM by genotype (WT mice, $7.8 \pm 0.2 \mathrm{~Hz}$; DS mice, $7.0 \pm 0.2 \mathrm{~Hz} ; p=$ $0.03, r=-0.8)$. Calibration: $\boldsymbol{A}, \boldsymbol{B}, 200 \mathrm{~ms}, 500 \mu \mathrm{V}$. Statistical analysis was performed by Wilcoxon-Mann-Whitney two-sample rank test $(p)$, and effect size is reported as rank-biserial correlation $(r) .{ }^{*} p<0.05,{ }^{* * *} p<0.005$. Spectral power in $\boldsymbol{C}$ and $\boldsymbol{E}$ was normalized by average power in a visually identified sleep period (see Materials and Methods).

\section{Results}

Spectral density analysis of characteristic

hippocampal rhythms

SPW-R features in DS mice were measured by implanting chronic fine-wire depth electrodes in dorsal CA1 proximal to stratum radiatum to simultaneously record ripple oscillations and SPW, maximal in the pyramidal cell layer and mid-stratum radiatum, respectively (DS mice, $n=7$; WT mice, $n=5$ ). Electrode locations were confirmed by characteristic LFP patterns and post hoc histology (Materials and Methods). Periods of activity (i.e., exploration, ambulation), quiet wakefulness, or rest (i.e., immobility, grooming), and non-rapid eye movement (NREM) sleep were identified off-line based on published approaches (Klausberger et al., 2003; Lapray et al., 2012; Materials and Methods) and analyzed separately. In both genotypes, characteristic state-dependent LFP patterns and transitions between states are easily identifiable by visual inspection. Periods of activity are characterized by distinctive theta-gamma oscillations with similar gross morphology (Fig. 1A), while periods of rest and sleep are characterized by irregular mixed-frequency activities (Fig. 1B).

To examine the range of LFP oscillation frequencies in each state, average power spectra describing the distribution of LFP power by frequency components were determined separately for periods of exploration, rest, and sleep. During exploration, there is a small but significant reduction in theta-band $(5-12 \mathrm{~Hz})$ power and frequency in DS mice (Fig. $1 E, F$; WT mice, $7.8 \pm 0.2$ $\mathrm{Hz}$; DS mice, $7.0 \pm 0.2 \mathrm{~Hz} ; p=0.03, r=-0.8$ ), while the distri- bution of gamma-band frequency $(30-80 \mathrm{~Hz})$ is broad and largely overlaps WT littermate values (Fig. $1 E$ ). More significantly, during periods of NREM sleep, peak ripple-band power $(100-260 \mathrm{~Hz})$ is slower in DS mice (Fig. 1C,D; WT mice, $170 \pm 4$ $\mathrm{Hz}$; DS mice, $141 \pm 4 \mathrm{~Hz} ; p=0.003, r=-1$ ), and, notably, ripple-band spectral power is also reduced (Fig. 1C). These findings demonstrate that Scnla mutation in DS mice is sufficient to alter but not abolish characteristic hippocampal network activity, particularly in the ripple frequency band during periods of sleep and rest, and previous work suggests that it may be sufficient to impair spatial learning and memory (Girardeau et al., 2009; Jadhav et al., 2012).

\section{Internal ripple frequency of SPW-R determined by detection} of individual SPW-R complexes

The most significant impacts of Scn 1a mutation were in the ripple band, and ripple formation critically depends on fast-spiking interneuron activity, which is impaired in DS mice. Additional evaluation of SPW-R was performed to determine whether the internal ripple frequency of SPW-R complexes is reduced in DS mice and to identify other SPW-R factors contributing to reduced ripple-band power. SPW-R morphology is grossly preserved in DS mice (Fig. 1A), but alterations in amplitude and more subtle changes in morphology could bias ripple detection based on commonly used automated, threshold-based algorithms. Therefore, transient increases in normalized instantaneous ripple-band power were used for individual SPW-R event detection in LFP recordings off-line, allowing for direct comparison between mice and accounting for variability in the recording site (Fig. 2A; Klausberger et al., 2003; Lapray et al., 2012; Materials and Methods).

Following detection of individual SPW-R, power spectral analysis was repeated for periods of sleep with and without SPW-R detections. Ripple-band power is only minimally increased in power spectra generated from periods of sleep excluding detected SPW-R (Fig. 2B), confirming that SPW-R complexes account for the preponderance of increased ripple-band (100$260 \mathrm{~Hz}$ ) spectral power and that significant numbers of SPW-R complexes were not missed by automated ripple detection. In epileptic hippocampus, ripples can evolve into epileptic pHFOs, which would result in fewer SPW-R detections (Gulyás and Freund, 2015). The rate of interictal epileptic activity in DS mice is greatest during periods of sleep (Kalume et al., 2015). To screen for high-frequency $(260-600 \mathrm{~Hz})$ activity characteristic of pHFOs, the ratio of average spectral density for periods of sleep with and without ripples was determined. No significant pHFO $(260-600 \mathrm{~Hz})$ power is present during or between ripples, thus SPW-R detection is not biased by the transformation of ripples into pHFOs (Fig. 2D).

Internal ripple frequency was estimated for each detected SPW-R complex by averaging instantaneous frequency from three or more intertrough intervals around the center ripple trough. Individual ripple frequency estimates were then used to determine the mean ripple frequency for each mouse during sleep and rest, and were averaged by genotype. DS mice show slowed internal ripple frequency in both sleep and rest states (Fig. $2 C$; sleep: WT mice, $160 \pm 1 \mathrm{~Hz}$; DS mice, $130 \pm 3 \mathrm{~Hz}$; $p=0.003$, $r=-1$; rest: WT mice, $157 \pm 3 \mathrm{~Hz}$; DS mice, $130 \pm 2, \mathrm{~Hz} ; p=$ $0.003, r=-1$ ), which is consistent with the shift in ripple-band frequency observed in power spectra, confirming that this shift is due to slowed internal ripple frequency. 
A

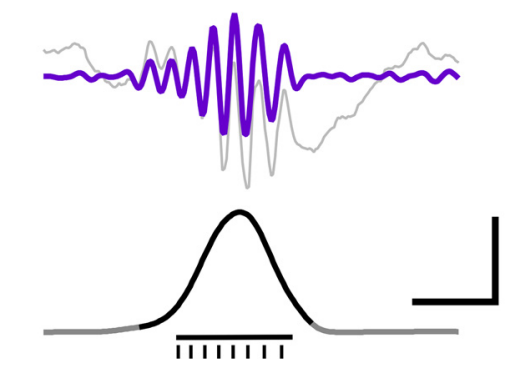

C

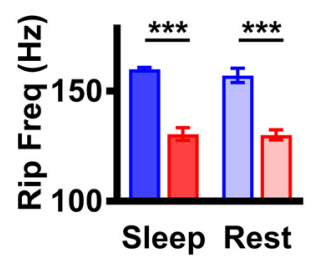

B
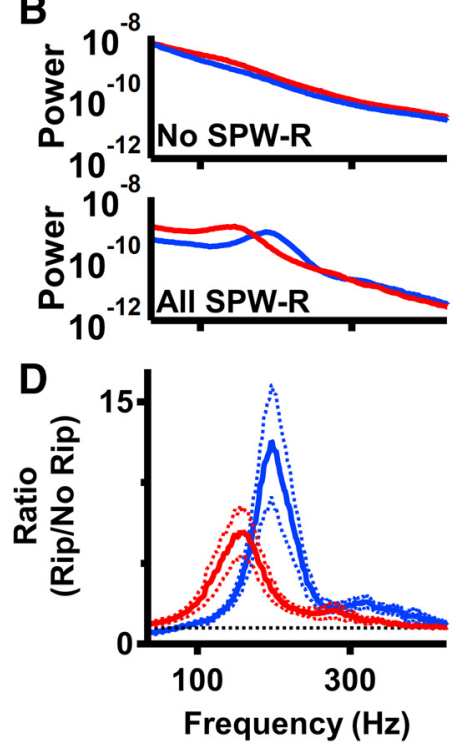

Figure 2. Average features from individually detected SPW-Rs confirm slowed frequency in DS mice. $\boldsymbol{A}$, Representative example of ripple detection. Top, Wide-band filtered "raw" traces in gray, ripple band-filtered LFP $(100-260 \mathrm{~Hz})$, shown in purple. Bottom, Normalized instantaneous power in the ripple band-filtered LFP $(100-260 \mathrm{~Hz}$, gray trace). Start and end of candidate SPW-R region is defined as the time of 0.5 SD threshold crossings, and ripple duration is denoted by the black bar under instantaneous ripple-band power (also in black); hash marks below indicate individual ripple trough detections. Ripples with peak power $>2$ SDs were accepted. Calibration: $50 \mathrm{~ms}, 100 \mu \mathrm{V}$ or 10 SDs from mean. B, Average spectrograms of periods of time without SPW-Rs (top) and all detected SPW-Rs (bottom; blue: WT, $n=5$; red: DS, $n=7$ ). The deviation from linear seen in SPW-R-containing time periods is absent in the no-SPW-R spectrograms, indicating that the majority of individual SPW-Rs have been detected and account for nearly all of the power seen in that range in the whole-LFP recordings. C, Average ripple frequency (sleep: WT mice, $160 \pm 1 \mathrm{~Hz}$; DS mice, $130 \pm 3 \mathrm{~Hz} ; p=0.003, r=-1$; rest: WT mice, $157 \pm 3 \mathrm{~Hz}$; DS mice, $130 \pm 2, \mathrm{~Hz} ; p=0.003, r=-1)$. Statistical analysis was performed by Wilcoxon-Mann-Whitney two-sample rank test ( $p$ ), and effect size is reported as rank-biserial correlation $(r)$. ${ }^{* * *} p<0.005$. D, Ratio of average frequency spectrogram by genotype during periods of sleep containing SPW-R detections vs periods of time without. Values $>1$ (black dashed line) indicate that frequencies are enriched in SPW-R-containing windows of time. Genotype average \pm SEM (blue: WT, $n=5$; red: DS, $n=7$ ).

Potential factors contributing to reduced ripple-band power, rate of SPW-R occurrence, and duration of ripples

In addition to slowed internal frequency, ripple-band spectral power is reduced in DS mice (Fig. 1C), which could be due to less frequent, shorter, or lower-amplitude SPW-R complexes. Average SPW-R morphology is similar in each genotype, estimated by averaging LFP aligned in time on the middle trough of each detected SPW-R complex (Fig. 3A). The rate of SPW-R occurrence, estimated in each mouse as the number of detected SPW-Rs divided by the total time in each state, is decreased in both sleep and rest states in DS mice (Fig. $3 B$; sleep: WT mice, $0.87 \pm 0.12$ SPW-R/s; DS mice, $0.47 \pm 0.08 \mathrm{SPW}-\mathrm{R} / \mathrm{s} ; p=0.048, r=-0.7$; rest: WT mice, $0.80 \pm 0.15 \mathrm{SPW}-\mathrm{R} / \mathrm{s}$; DS mice, $0.38 \pm 0.06 \mathrm{SPW}-$ $\mathrm{R} / \mathrm{s} ; p=0.03, r=-0.8)$. As previously discussed, the decreased occurrence of SPW-Rs in DS mice is not explained by pHFO (Fig. $2 D)$. Similar proportions of SPW-Rs occur in burst complexes (Fig. $3 C$; sleep: WT mice, $0.37 \pm 0.05$; DS mice, $0.31 \pm 0.05 ; p=$ $0.6, r=-0.2$; rest: WT mice, $0.38 \pm 0.08$; DS mice, $0.27 \pm 0.05$; $p=0.3, r=-0.4$ ), defined as the occurrence of two or more SPW-Rs separated by $<100 \mathrm{~ms}$ (Csicsvari et al., 2007; Schlingloff et al., 2014), and the reduced rate of occurrence is not due to fewer SPW-Rs per burst.

The duration of the ripple component of each SPW-R complex is defined as the time between the first and last detected troughs and shows a trend toward reduced duration in DS mice, but does not reach significance (Fig. $3 D$; sleep: WT mice, $39.2 \pm$ $2.1 \mathrm{~ms}$; DS mice, $38.1 \pm 1.0 \mathrm{~ms} ; p=1, r=0.03$; rest: WT mice, $38.5 \pm 1.8 \mathrm{~ms}$; DS mice, $36.4 \pm 1.1 \mathrm{~ms} ; p=0.4, r=-0.3)$.
Therefore, decreased ripple-band power is not the result of reduced ripple duration. Consistent with slower internal ripple oscillations (Fig. 2C) and similar ripple duration (Fig. $3 D$ ), the number of oscillation cycles per ripple, estimated by the number of troughs detected, is reduced in DS mice (Fig. 3E; sleep: WT mice, $7.3 \pm 0.3$; DS mice, $5.8 \pm 0.2, p=$ 0.003; $r=-1$; rest: WT mice, $7.5 \pm 0.4$; DS mice, $5.6 \pm 0.2 ; p=0.003, r=-1$ ). Although there is a trend toward lower individual ripple power in DS mice (Fig. 3F; sleep: WT mice, $9.1 \pm 1.8$; DS mice, $7.2 \pm 0.4 ; p=0.9, r=-0.1$; rest: WT mice, $9.4 \pm 1.0$; DS mice, $7.3 \pm 1.1 ; p=$ $0.1, r=-0.6)$, the difference is not significant. Together, these data confirm that decreased ripple-band power (Fig. 1C) is not attributable to reduced ripple duration or smaller individual ripple amplitudes, but rather to results from a reduced rate of SPW-R occurrence.

\section{Dependence of ripple internal \\ frequency, power, and timing on SPW characteristics}

Within CA1, SPW-Rs are generated locally in response to CA3 pyramidal cellmediated SPW excitation, and there is a positive correlation between SPW amplitude and internal ripple oscillation frequency (Ponomarenko et al., 2004; Nguyen et al., 2009; Sullivan et al., 2011; Stark et al., 2014). Therefore, slower ripples in DS mice could be explained by lower-amplitude SPW. To determine whether changes in SPW amplitude contribute to slowed internal ripple frequency in DS mice, the corresponding SPW was identified in bandpass-filtered (10-20 Hz) LFPs for each detected ripple, and normalized instantaneous power in the ripple $(100-260 \mathrm{~Hz})$ and SPW $(10-20 \mathrm{~Hz})$ frequency bands was compared with control for differences in signal-to-noise ratio across recordings sites and mice (Materials and Methods). Ripple power increases with SPW power across a comparable range and with a similar distribution in both genotypes (Fig. $4 A, B$ ), and at equivalent SPW power, DS ripple frequencies are always slower (Fig. 4C). Similarly, the number of ripple troughs in each SPW-R complex is positively correlated with SPW power in both genotypes but is always lower in DS mice (Fig. 4D).

During an SPW-R complex, the frequency of the internal ripple oscillation is highest at sharp wave onset and decreases thereafter (Ponomarenko et al., 2004; Nguyen et al., 2009; Sullivan et al., 2011; Stark et al., 2014). Delay in the timing of rippling relative to SPW could contribute to reduced internal ripple frequency in DS mice. To determine how ripple features vary over time during an SPW, peri-SPW-R average responses were calculated for each detected SPW-R by averaging $\pm 40 \mathrm{~ms}$ windows of LFP aligned on the ripple trough closest to the SPW, an average for each mouse was calculated, and grand average responses were then determined by genotype (Fig. 5A). Average ripple (100-260 $\mathrm{Hz})$ and SPW (10-20 Hz) bandpass-filtered responses have a similar time course and amplitude, while intervals between ripple troughs are further apart in the DS grand average, reflecting re- 

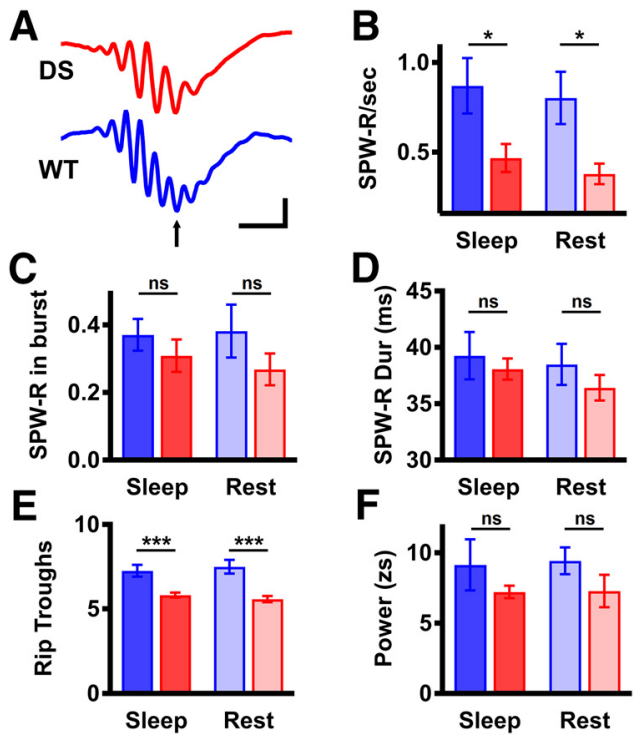

Figure 3. Average features from individually detected SPW-Rs confirm a reduced occurrence in DS mice. Average SPW-R features by genotype in rest and sleep states. $A$, CA1 LFP average SPW-Rs by genotype (blue: WT mice, $n=5$; red: DS mice, $n=7$ ) during periods of sleep and rest. SPW-Rs were aligned on the ripple trough nearest to the SPW trough (arrow). Calibration: $20 \mathrm{~ms}, 50 \mu \mathrm{V}$. B, Average rate of SPW-R occurrence expressed as SPW-R/s (sleep: WT mice, $0.87 \pm 0.12 ;$ DS mice, $0.47 \pm 0.08 ; p=0.048, r=-0.7$; rest:WT mice, $0.80 \pm 0.15$; DS mice, $0.38 \pm 0.06 ; p=0.03, r=-0.8)$. C, Proportion of SPW-Rs occurring within a SPW-R burst complex (sleep: WT mice, $0.37 \pm 0.05$; DS mice, $0.31 \pm 0.05 ; p=0.6, r=-0.2$; rest: WT mice, $0.38 \pm 0.08 ; D S$ mice, $0.27 \pm 0.05 ; p=0.3, r=-0.4$ ). D, Average SPW duration (sleep: WT mice, $39.2 \pm 2.1 \mathrm{~ms} ;$ DS mice, $38.1 \pm 1.0 \mathrm{~ms} ; p=1, r=0.03$; rest: WT mice, $38.5 \pm 1.8$ $\mathrm{ms}$; DS mice, $36.4 \pm 1.1 \mathrm{~ms} ; p=0.4, r=-0.3$ ). $\boldsymbol{E}$, Average number of troughs (cycles) per ripple (sleep: WT mice, $7.3 \pm 0.3 ; \mathrm{DS}$ mice, $5.8 \pm 0.2 ; p=0.003, r=-1$; rest:WT mice, $7.5 \pm$ $0.4 ;$ DS mice, $5.6 \pm 0.2 ; p=0.003, r=-1$ ). $\boldsymbol{F}$, Average ripple power (sleep: WT mice, $9.1 \pm$ 1.8; DS mice, $7.2 \pm 0.4 ; p=0.9, r=-0.08$; rest: WT mice, $9.4 \pm 1.0 ;$ DS mice, $7.3 \pm 1.1 ; p=$ $0.1, r=0.6)$. Statistical analysis was performed using the Wilcoxon-Mann-Whitney twosample rank test $(p)$, and effect size is reported as rank-biserial correlation $(r) .{ }^{*} p<0.05$; ${ }^{* * *} p<0.005 ;$ ns, not significant.
A

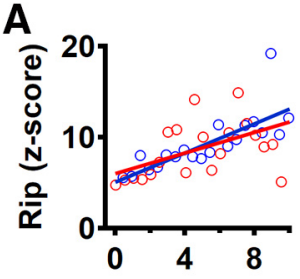

B

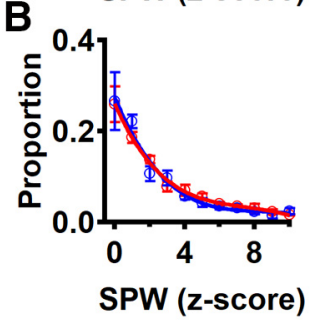

C

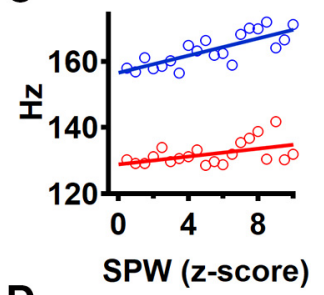

D

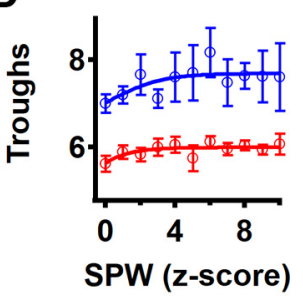

WT - DS
Figure 4. DS ripple frequency is reduced independent of sharp wave power. $A$, In both WT (blue, $n=5$ ) and DS (red, $n=7$ ) mice, ripple power increases similarly with SPW power. Genotype average ripple power by SPW power (bin size, 0.5 SD from mean). B, Distribution of SPW power by genotype. $\boldsymbol{C}$, Average ripple frequency by SPW power for each genotype. $\boldsymbol{D}$, Average number of ripple troughs by SPW amplitude for each genotype.

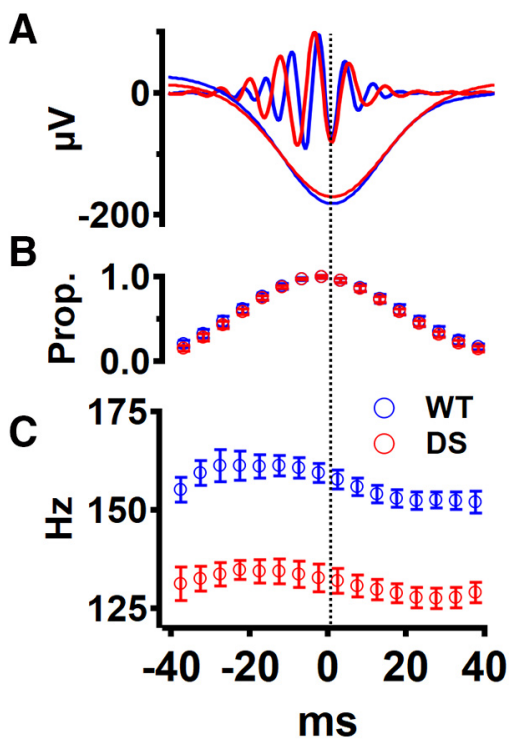

Figure 5. Timing of ripple relative to SPWs is maintained in DS mice. Average ripple features by time aligned on the ripple trough nearest the SPW trough. Blue: WT, $n=5$; red: DS, $n=7$. $A$, Averages of LFP recordings bandpass filtered for SPWs $(10-20 \mathrm{~Hz})$ or ripples $(100-260 \mathrm{~Hz})$. Note that rippling is more prominent on the falling phase of the SPW peaking before the SPW trough. $\boldsymbol{B}$, Proportion of detected SPW- $R$ complexes in which rippling is present at times relative to the SPW trough. $C$, Average instantaneous ripple frequency. Average ripple frequency is highest early in the ripple and decreases throughout, as previously described. The time course of frequency decay is similar in both genotypes, although the absolute frequency remains lower in DS ripples throughout.

duced overall internal ripple frequency (Fig. 5A). To characterize the probability of ripple occurrence relative to SPW, the presence of rippling was determined in $5 \mathrm{~ms}$ bins. In both genotypes, the probability of rippling is greatest just before the SPW trough, falling symmetrically with time on either side (Fig. 5B). Average instantaneous frequency, determined from intertrough intervals in $5 \mathrm{~ms}$ bins (Fig. $5 \mathrm{C}$ ), is fastest at SPW onset and slows over time (WT mice, $\sim 160-150 \mathrm{~Hz}$; DS mice, $\sim 135-125 \mathrm{~Hz}$ ). Although the average SPW amplitude is similar, in DS mice, internal ripple frequency is consistently slower at all latencies from the SPW trough (Fig. 5C), implicating the impairment of intrinsic CA1 ripple-generating mechanisms rather than a systematic reduction in SPW amplitude in the reduced internal frequency of ripples.

Impact of decreased sodium conductance on oscillatory frequency in a simple in silico interneuron model

The preceding in vivo studies implicate impaired ripplegenerating mechanisms in CA1 as the mediator of slowed internal ripple frequency in DS mice. To examine whether reduced sodium conductance and impaired excitability of fast-spiking interneurons in DS mice alone could be sufficient to affect the synchronization and pacing of network activity, a simple in silico model was created. The model contains 100 fully interconnected conductance-based interneurons. Modified Hodgkin-Huxley neurons, as detailed previously (Wang and Buzsáki, 1996), were chosen for their fast-spiking behavior. Pyramidal cells (Fig. 6A, gray cells) were not included because the activation of fastspiking interneurons alone, particularly parvalbumin basket cells, is sufficient to pace network activity at ripple frequency (Schlingloff et al., 2014; Stark et al., 2014), and ripple frequency timing does not require pyramidal cell activation or CA1 pyramidal cell excitation onto local interneurons (Rácz et al., 2009; Stark et al., 2014). Moreover, the mutation of Scn1a in excitatory neu- 

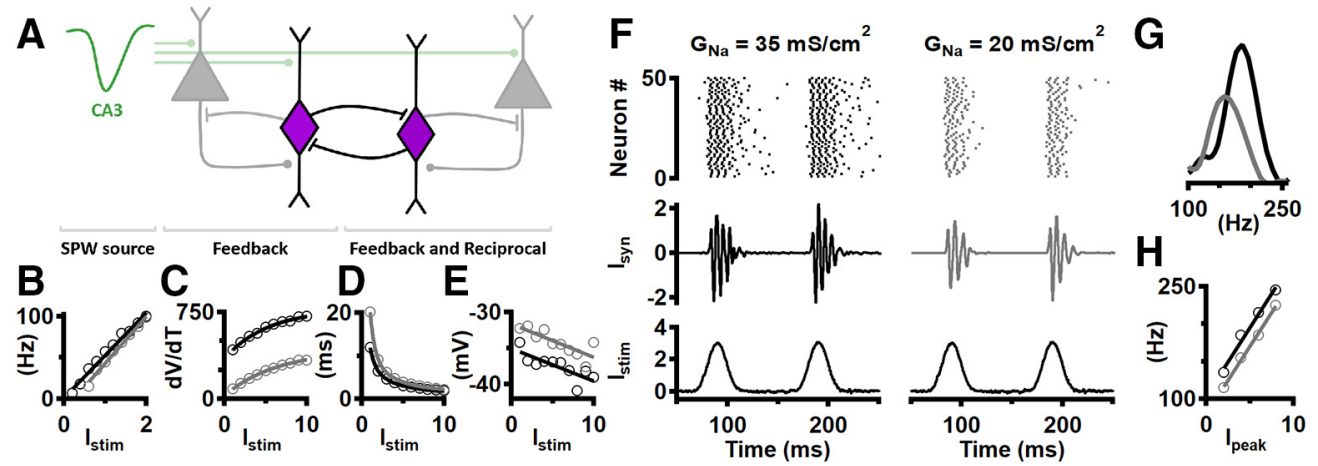

Figure 6. Reduced sodium conductance decreases cell excitability and slows the frequency of simulated ripples in an in silico fully connected interneuron network model. $\boldsymbol{A}$, Summary of proposed network models of SPW-R generation in CA1 following SPW invasion from CA3 (green). Direct stimulation of pyramidal (gray) and inhibitory cells (purple) is proposed in the feedback model (Schlingloff et al., 2014). Feedback and reciprocal stimulation proposes pyramidal cells (gray) are directly stimulated by the SPW and then indirectly stimulates an interconnected interneuron network (purple; Stark et al., 2014). The current model consists of the common element in both models: interconnected IN, shown in purple. B, Firing rate increases with increasing current. Constant Gaussian noise was added to the input $\left(S D=0.5 \mu \mathrm{A} / \mathrm{cm}^{2}\right) . C, D$, Maximal first derivatives of spike membrane potential are decreased $(\boldsymbol{C})$ and time to first spike after onset of constant current is increased with lowered sodium conductance $(\boldsymbol{D})$. $\boldsymbol{E}$, Spiking threshold was measured at the point of the maximal second derivative during a potential upswing. $\boldsymbol{F}$, Top, Neuron \#: neural networks of 50 of the 100 fully connected Wang-Buzsáki interneurons with high sodium $\left(G_{\mathrm{Na}}=35 \mathrm{mS} / \mathrm{cm}^{2} ;\right.$ black $)$ and low sodium $\left(G_{\mathrm{Na}}=20 \mathrm{mS} / \mathrm{cm}^{2} ;\right.$ gray) with shunting inhibition $\left(E_{\text {syn }}=-55\right)$ and fast synapses $\left(\tau_{\text {syn }}=2 \mathrm{~ms}\right.$ ). Middle, $I_{\text {syn }}$ : bandpass-filtered synaptic currents (second-order digital Butterworth filter, $100-300 \mathrm{~Hz}$ ) show shorter, slower ripples in the low sodium conductance network (red lines). Bottom, $I_{\text {stim }}$ is composed of periodic Gaussians (FWHM $=20 \mathrm{~ms}$ ) with superimposed constant Gaussian-filtered ( $\tau=1 \mathrm{~ms}$ ) white noise $\left(S D=0.5 \mu \mathrm{A} / \mathrm{cm}^{2}\right)$ repeated every $100 \mathrm{~ms}$. G, Peak ripple-band power in normal $G_{\mathrm{Na}}$ (black) model interneurons and lower $G_{\mathrm{Na}}$ peak ripple-band power (gray). Both are comparable to peak ripple power in vivo (Fig. 1C). $\boldsymbol{H}$, Lower sodium conductance $\left(G_{\mathrm{Na}}=20 \mathrm{mS} / \mathrm{cm}^{2} ;\right.$ gray) leads to slower intrinsic ripple frequencies (mean, $22.5 \mathrm{~Hz}$ slower) compared with higher sodium conductance $\left(G_{\mathrm{Na}}=35 \mathrm{mS} / \mathrm{cm}^{2} ;\right.$ black line) over a range of input stimuli. Stimulus noise, $\mathrm{SD}=0.5 \mu \mathrm{A} / \mathrm{cm}^{2}$.

rons does not cause DS symptoms in mice (Ogiwara et al., 2013). The stimulus to the network was composed of Gaussian-shaped positive currents simulating SPW-mediated excitation, repeated every $100 \mathrm{~ms}$, as might occur during an SPW-R burst complex (Fig. $6 F$, bottom, $I_{\text {stim }}$ (input current stimulus); Csicsvari et al., 2000, 2007; Schlingloff et al., 2014). Model synapses are fast $\left(\tau_{\text {syn }}\right.$ $=2 \mathrm{~ms})$ and strong $\left(G_{\mathrm{syn}}=1 \mathrm{mS} / \mathrm{cm} 2\right)$, and the reversal potential $\left(E_{\text {syn }}=-55 \mathrm{mV}\right.$ ) leads to shunting inhibition (Bartos et al., 2007). To compare in silico model behavior to in vivo LFP ripples, mean synaptic currents averaged from all synapses (Fig. $6 F, I_{\text {syn }}$ ) and mean synaptic current spectral power were determined in each model (Fig. $6 G$ ).

Sodium current in GABAergic interneurons is significantly reduced in DS mouse models (Yu et al., 2006), and comparisons were therefore made between models with normal sodium conductance $\left(G_{\mathrm{Na}}=35 \mathrm{mS} / \mathrm{cm}^{2}\right)$ and reduced sodium conductance $\left(G_{\mathrm{Na}}=20 \mathrm{mS} / \mathrm{cm}^{2}\right)$ chosen to approximate the electrophysiologic properties of INs previously reported in WT and DS mice in vitro (Yu et al., 2006; Ogiwara et al., 2007; Tai et al., 2014; Rubinstein et al., 2015). When single-cell properties were examined in isolation, decreased sodium conductance led to reduced excitability in the spike-generating mechanism (Fig. $6 B-E$ ). Specifically, low $G_{\mathrm{Na}}$ neurons had a decreased rate of firing (Fig. $6 B$ ), more slowly moving membrane potential during firing (Fig. $6 \mathrm{C}$ ), a delay in time to first spike (Fig. $6 D$ ), and elevated spike threshold (Fig. 6E).

In response to repeated Gaussian excitatory stimuli of sufficient strength (Fig. $6 F, I_{\text {stim }}$ ), model neurons from both the high $G_{\mathrm{Na}}$ (black) and low $G_{\mathrm{Na}}$ (DS, gray) model networks fire repeatedly with synchronized spike timing (Fig. $6 F$, top), leading to inhibitory synaptic currents that fluctuate at high frequencies $\left(100-300 \mathrm{~Hz}\right.$ ) during the stimulus pulse (Fig. $\left.6 F, I_{\text {syn }}\right)$. Reducing sodium conductance in the model slows the frequency of oscillation (Fig. $6 F$, $\mathrm{I}_{\text {syn }}$ oscillations: black, high $G_{\mathrm{Na}}$; gray, low $G_{\mathrm{Na}}$ ), generating a shift in spectral power to slower frequencies (Fig. $6 G$ ) similar to those observed in spectra from in vivo LFP ripples (Fig. 1C). In both models, oscillation frequencies vary with the amount of excitatory input stimulus the network receives (Fig. $6 \mathrm{H}$ ). However, for any given amount of input excitation, frequencies from the low $G_{\mathrm{Na}}$ network are $\sim 20 \mathrm{~Hz}$ less (Fig. $6 \mathrm{H}$ ), with the difference in frequency varying in direct proportion to the difference in $G_{\mathrm{Na}}$ between the networks (data not shown).

\section{Discussion}

Impaired SPW-Rs likely contribute to cognitive deficits in DS mice

Neuropsychological tests indicate that contextual memory is impaired in DS (Chieffo et al., 2011a; Guerrini and Falchi, 2011; Ragona et al., 2011), and there is an increased rate of accidental injury and death, possibly reflecting an inability to recall and avoid dangerous situations (Skluzacek et al., 2011; Shmuely et al., 2016). Improvement in cognitive function is an important treatment goal in DS, but specific pathophysiologic mechanisms linking SCN1A haploinsufficiency and cognitive deficits are lacking. This study demonstrates that $S c n 1 a$ haploinsufficiency, the most frequent cause of DS, is sufficient to slow hippocampal theta frequency, reduce SPW-R occurrence, and slow internal ripple frequency, likely contributing to spatial memory impairment in DS mice (Han et al., 2012a; Ito et al., 2013).

The rate of SPW-R occurrence is decreased by $\sim 30 \%$ in DS mice, and a similar magnitude of electrical or optogenetic disruption of SPW-R impairs spatial learning and memory in rodents (Girardeau et al., 2009; van de Ven et al., 2016). Here, internal ripple frequency is also slowed by $\sim 30 \mathrm{~Hz}$ in DS mice, resulting in fewer ripple cycles per SPW-R complex. Each ripple cycle encodes unique information, and a reduction of the number of cycles decreases the information encoded by each SPW-R complex. During learning, SPW-R amplitude and frequency increase (Ponomarenko et al., 2008) and optogenetic prolongation of endogenous SPW-R enhances learning (Fernández-Ruiz et al., 2019), directly implicating SPW-R properties as a physiologic learning mechanism. In light of those studies, slowed internal ripple frequency in DS mice forges a likely causal mechanistic 
link between the mutation of Scnla and impaired spatial memory in DS.

SPW-R impairment has been reported in aging-related neurologic conditions (Booth et al., 2016; Gillespie et al., 2016; Wiegand et al., 2016). Reduced SPW-R occurrence and slowed internal ripple frequency correlate with age-related cognitive decline (Gillespie et al., 2016; Wiegand et al., 2016), and the restoration of internal ripple frequency alone is sufficient to improve cognitive function in an Alzheimer's model (Gillespie et al., 2016). Additionally, toxin-mediated blockade of signaling between CA3 and CA1 has no impact on the number of SPW-R occurrences, but slows internal ripple frequency and impairs spatial memory (Nakashiba et al., 2009).

Conversely, pHFOs $(250-600 \mathrm{~Hz})$ have been reported in many epileptic conditions and animal models (Bragin et al., 2002; Jefferys et al., 2012; Valero et al., 2017). The emergence of pHFOs may result from a failure of SPW-R formation following an increase in internal frequency (Valero et al., 2017). This mechanism stands apart from the findings presented here, as DS mice show a slowing of SPW-R intrinsic frequency, underscoring the specificity of the impairment of GABAergic IN function in DS. Studies in a schizophrenia mouse model report an increased SPW-R rate and internal frequency with accompanying memory impairment but attribute the impairment to disordered place cell firing (Suh et al., 2013). Further studies in DS are required to assess the fidelity of place cell firing; however, the present study directly implicates both reduced SPW occurrence and slowed internal ripple frequency in spatial memory impairment in DS.

\section{Scn 1a mutation in DS mice reduces the occurrence of SPW-R with no change in the power and morphology of SPW}

SPW-R complexes occur less frequently in DS mice. However, both the distribution of SPW power (Fig. 4), a proxy for amplitude or strength, and SPW morphology (Fig. 5), determined by the timing and recruitment of $\mathrm{CA} 3$ pyramidal cells, are unchanged. These findings indicate that Scnla mutations in DS mice selectively impair mechanisms controlling SPW initiation but have little impact on mechanisms controlling pyramidal cell activity once SPWs are initiated, consistent with unchanged pyramidal cell firing in DS mice (Yu et al., 2006; Tai et al., 2014). GABAergic INs, the cell type most impacted by Scn 1 a mutation in DS (Yu et al., 2006; Ogiwara et al., 2007), are critical to the recruitment and timing of pyramidal cell activity, and both optogenetic (Stark et al., 2013; Schlingloff et al., 2014) and electrical (Ellender and Paulsen, 2010) manipulations of GABAergic signaling impact the rate of SPW occurrence (Buzsáki, 2015).

Pyramidal cells in CA3 are highly interconnected, and activity in a few pyramidal cells can precipitate an avalanche of activity through reciprocal excitation (Schlingloff et al., 2014). A brief period of rebound excitation following parvalbumin interneuron (PVIN)-mediated inhibition paces the synchronous activity of pyramidal cells (Ellender and Paulsen, 2010; Stark et al., 2013). SPWs arise stochastically as a population burst in CA3, in part under the control of local and projection GABAergic INs, including fast-spiking, long-range GABAergic projection INs in the medial septum diagonal band (MSDB; Vandecasteele et al., 2014; Takács et al., 2018). Targeted siRNA-mediated knockdown of Scnla in MSDB decreases the excitability of GABAergic projection cells, slows SPW occurrence, and impairs spatial memory (Bender et al., 2016). MSBD-specific Scn1a knockdown also slows hippocampal theta frequency during exploratory activity (Bender et al., 2013, 2016), similar to the reduced theta frequency observed in DS mice (Fig. 1). Together, these results suggest that global Scnla haploinsufficiency could contribute to reduced SPW generation through impaired PVIN firing and reduced GABAergic signaling from MSDB.

\section{Reduced excitability of GABAergic interneurons is the likely cause of slowed internal ripple frequency}

Overlapping distributions of both WT and DS SPWs and ripple power (Fig. $4 A, B$ ), and the positive correlation between ripple frequency and SPW power (Fig. 4C), implicate local CA1 mechanisms rather than reduced excitatory drive as the source of the slowed ripple frequency. When a CA3-generated SPW invades $\mathrm{CA1}$, it induces local high-frequency oscillations (ripples; Ylinen et al., 1995). Within CA1, PVINs fire on nearly every ripple cycle to synchronize local pyramidal cells and to generate the alternating pattern of excitatory and inhibitory activity characteristic of ripples. The summation of currents generated by this coordinated pyramidal cell firing, entrained to the inhibitory frequency, allows network activity to be visualized by LFP (Klausberger and Somogyi, 2008; Chiovini et al., 2014; Gan et al., 2017). Consistent with this mechanism, optogenetic stimulation of CA1 PVINs generates ripple frequency oscillation (Schlingloff et al., 2014), while optogenetic silencing of PVINs leads to impairment (Stark et al., 2014). In vivo, Scnla-related reduction in PVIN firing impairs spatial memory (Rubinstein et al., 2015; Tatsukawa et al., 2018).

The interplay of excitatory and inhibitory cells underlying the pacing of SPW-R frequency in CA1 are unclear (Donoso et al., 2018). One theory posits that both excitatory and inhibitory cells receive direct SPW stimulation and that the synaptic interaction between them sets the frequency of the ripple oscillation (Fig. 6A, "feedback inhibition"; Schlingloff et al., 2014). However, ripple frequency oscillations persist when excitatory synapses onto inhibitory neurons are blocked, implicating intact IN signaling in setting oscillation timing (Rácz et al., 2009). A variation of this model hypothesizes that pyramidal cells receive direct inputs from $\mathrm{CA} 3$, which in turn serve as the excitatory drive to an interconnected IN network (Fig. 6A, "feedback and reciprocal"; Stark et al., 2014). Following optogenetic stimulation of PVINs, sparsely active pyramidal cells are entrained to the PV firing frequency in the absence of a detectable LFP ripple (Stark et al., 2014). From these and other models, a picture of SPW-R dynamics emerges in which inhibitory networks constrain and pace the activity of local pyramidal cells and pyramidal cell activity serves as a "readout" of this network pacing.

Here, intrinsic SPW-R frequency is slowed in DS mice (Figs. $1 C, D, 2 C$ ), while the gross morphology (Fig. $3 A$ ) and power (Fig. $3 F$ ) of the detected SPW-Rs remain unchanged. These data are consistent with GABAergic INs as the class of neurons most affected by Scnla mutation and in setting the timing of network oscillation. A simple in silico model of the common element in these circuits, the interconnected INs (Donoso et al., 2018), was used to explore whether a reduction in the sodium current of INs would alone be sufficient to cause the observed SPW-R impairments seen in vivo. In vitro, the firing rate of DS PVINs is slower for any given stimulus level (Tai et al., 2014), and model interneurons were comparably impacted (Fig. $6 B-E$ ). Similar to in vivo data, model oscillation frequencies increase with excitatory input (Fig. 6G), and oscillation frequencies from the low-sodium conductance network are always $\sim 20 \mathrm{~Hz}$ lower than neurons with normal sodium conductance (compare Figs. 6G, 4C). Thus, this simple, straightforward model demonstrates that reduced sodium conductance in model cells is sufficient to slow both IN 
firing and model ripples comparable to the decreases in internal ripple frequency observed in DS mice.

The altered SPW-R properties identified in this study provide a specific pathophysiologic mechanism connecting the mutation of Scn1a and its resulting cellular deficits in inhibitory neuron function with whole-animal behavioral impairment in DS mice. Furthermore, these SPW-R properties may be useful biomarkers for cognitive impairment in DS, and the unique mechanism by which SPW-Rs are altered in DS mice, Scnla mutation-related decrease in IN excitability, may be a potential therapeutic target to improve cognitive function in DS patients.

\section{References}

Bartos M, Vida I, Jonas P (2007) Synaptic mechanisms of synchronized gamma oscillations in inhibitory interneuron networks. Nat Rev Neurosci 8:45-56.

Bender AC, Natola H, Ndong C, Holmes GL, Scott RC, Lenck-Santini PP (2013) Focal Scnla knockdown induces cognitive impairment without seizures. Neurobiol Dis 54:297-307.

Bender AC, Luikart BW, Lenck-Santini PP (2016) Cognitive deficits associated with Nav1.1 alterations: involvement of neuronal firing dynamics and oscillations. PLoS One 11:e0151538.

Booth CA, Witton J, Nowacki J, Tsaneva-Atanasova K, Jones MW, Randall AD, Brown JT (2016) Altered intrinsic pyramidal neuron properties and pathway-specific synaptic dysfunction underlie aberrant hippocampal network function in a mouse model of tauopathy. J Neurosci 36:350-363.

Bragin A, Engel J Jr, Wilson CL, Fried I, Buzsáki G (1999) High-frequency oscillations in human brain. Hippocampus 9:137-142.

Bragin A, Mody I, Wilson CL, Engel J Jr (2002) Local generation of fast ripples in epileptic brain. J Neurosci 22:2012-2021.

Buzsáki G (2015) Hippocampal sharp wave-ripple: a cognitive biomarker for episodic memory and planning. Hippocampus 25:1073-1188.

Chieffo D, Battaglia D, Lettori D, Del Re M, Brogna C, Dravet C, Mercuri E, Guzzetta F (2011a) Neuropsychological development in children with Dravet syndrome. Epilepsy Res 95:86-93.

Chieffo D, Ricci D, Baranello G, Martinelli D, Veredice C, Lettori D, Battaglia D, Dravet C, Mercuri E, Guzzetta F (2011b) Early development in Dravet syndrome; visual function impairment precedes cognitive decline. Epilepsy Res 93:73-79.

Chiovini B, Turi GF, Katona G, Kaszás A, Pálfi D, Maák P, Szalay G, Szabó MF, Szabó G, Szadai Z, Káli S, Rózsa B (2014) Dendritic spikes induce ripples in parvalbumin interneurons during hippocampal sharp waves. Neuron 82:908-924.

Csicsvari J, Hirase H, Mamiya A, Buzsáki G (2000) Ensemble patterns of hippocampal CA3-CA1 neurons during sharp wave-associated population events. Neuron 28:585-594.

Csicsvari J, O’Neill J, Allen K, Senior T (2007) Place-selective firing contributes to the reverse-order reactivation of CA1 pyramidal cells during sharp waves in open-field exploration. Eur J Neurosci 26:704-716.

Dayan P, Abbott LF (2001) Theoretical neuroscience: computational and mathematical modeling of neural systems. Cambridge, MA: Massachusetts Institute of Technology.

Donoso JR, Schmitz D, Maier N, Kempter R (2018) Hippocampal ripple oscillations and inhibition-first network models: frequency dynamics and response to GABA modulators. J Neurosci 38:3124-3146.

Dravet C (2011) The core Dravet syndrome phenotype. Epilepsia 52 [Suppl 2]:3-9.

Ellender TJ, Paulsen O (2010) The many tunes of perisomatic targeting interneurons in the hippocampal network. Front Cell Neurosci 4:26.

Fernández-Ruiz A, Oliva A, Fermino de Oliveira E, Rocha-Almeida F, Tingley D, Buzsáki G (2019) Long-duration hippocampal sharp wave ripples improve memory. Science 364:1082-1086.

Gan J, Weng SM, Pernía-Andrade AJ, Csicsvari J, Jonas P (2017) Phaselocked inhibition, but not excitation, underlies hippocampal ripple oscillations in awake mice in vivo. Neuron 93:308-314.

Gerstner W, Kistler WM (2002) Spiking neuron models: single neurons, populations, plasticity. Cambridge, U.K.: Cambridge UP.

Gillespie AK, Jones EA, Lin YH, Karlsson MP, Kay K, Yoon SY, Tong LM, Nova P, Carr JS, Frank LM, Huang Y (2016) Apolipoprotein E4 causes age-dependent disruption of slow gamma oscillations during hippocampal sharp-wave ripples. Neuron 90:740-751.
Girardeau G, Zugaro M (2011) Hippocampal ripples and memory consolidation. Curr Opin Neurobiol 21:452-459.

Girardeau G, Benchenane K, Wiener SI, Buzsáki G, Zugaro MB (2009) Selective suppression of hippocampal ripples impairs spatial memory. Nat Neurosci 12:1222-1223.

Guerrini R, Falchi M (2011) Dravet syndrome and SCN1A gene mutation related-epilepsies: cognitive impairment and its determinants. Dev Med Child Neurol 53:11-15.

Gulyás AI, Freund TT (2015) Generation of physiological and pathological high frequency oscillations: the role of perisomatic inhibition in sharpwave ripple and interictal spike generation. Curr Opin Neurobiol 31: $26-32$.

Han S, Tai C, Westenbroek RE, Yu FH, Cheah CS, Potter GB, Rubenstein JL, Scheuer T, de la Iglesia HO, Catterall WA (2012a) Autistic-like behaviour in Scnla +/- mice and rescue by enhanced GABA-mediated neurotransmission. Nature 489:385-390.

Han S, Yu FH, Schwartz MD, Linton JD, Bosma MM, Hurley JB, Catterall WA, de la Iglesia HO (2012b) $\mathrm{Na}(\mathrm{V}) 1.1$ channels are critical for intercellular communication in the suprachiasmatic nucleus and for normal circadian rhythms. Proc Natl Acad Sci U S A 109:E368-E377.

Harris FJ (1978) On the use of windows for harmonic analysis with the discrete Fourier transform. Proc IEEE 66:51-83.

Hodgkin AL, Huxley AF (1952) A quantitative description of membrane current and its application to conduction and excitation in nerve. J Physiol 117:500-544.

Hu H, Gan J, Jonas P (2014) Interneurons. Fast-spiking, parvalbumin ${ }^{+}$ GABAergic interneurons: from cellular design to microcircuit function. Science 345:1255263.

Hutchison WD, Dostrovsky JO, Walters JR, Courtemanche R, Boraud T, Goldberg J, Brown P (2004) Neuronal oscillations in the basal ganglia and movement disorders: evidence from whole animal and human recordings. J Neurosci 24:9240-9243.

Ito S, Ogiwara I, Yamada K, Miyamoto H, Hensch TK, Osawa M, Yamakawa K (2013) Mouse with Nav1.1 haploinsufficiency, a model for Dravet syndrome, exhibits lowered sociability and learning impairment. Neurobiol Dis 49:29-40.

Jadhav SP, Kemere C, German PW, Frank LM (2012) Awake hippocampal sharp-wave ripples support spatial memory. Science 336:1454-1458.

Jefferys JG, Menendez de la Prida L, Wendling F, Bragin A, Avoli M, Timofeev I, Lopes da Silva FH (2012) Mechanisms of physiological and epileptic HFO generation. Prog Neurobiol 98:250-264.

Jeong J (2004) EEG dynamics in patients with Alzheimer's disease. Clin Neurophysiol 115:1490-1505.

Kalume F, Yu FH, Westenbroek RE, Scheuer T, Catterall WA (2007) Reduced sodium current in purkinje neurons from $\mathrm{Na}_{\mathrm{v}} 1.1$ mutant mice: implications for ataxia in severe myoclonic epilepsy in infancy. J Neurosci 27:11065-11074.

Kalume F, Westenbroek RE, Cheah CS, Yu FH, Oakley JC, Scheuer T, Catterall WA (2013) Sudden unexpected death in a mouse model of Dravet syndrome. J Clin Invest 123:1798-1808.

Kalume F, Oakley JC, Westenbroek RE, Gile J, de la Iglesia HO, Scheuer T, Catterall WA (2015) Sleep impairment and reduced interneuron excitability in a mouse model of Dravet syndrome. Neurobiol Dis 77:141-154.

Klausberger T, Somogyi P (2008) Neuronal diversity and temporal dynamics: the unity of hippocampal circuit operations. Science 321:53-57.

Klausberger T, Magill PJ, Márton LF, Roberts JD, Cobden PM, Buzsáki G, Somogyi P (2003) Brain-state- and cell-type-specific firing of hippocampal interneurons in vivo. Nature 421:844-848.

Koch C (1999) Biophysics of computation: information processing in single neurons. Oxford, U.K.: Oxford UP.

Lapray D, Lasztoczi B, Lagler M, Viney TJ, Katona L, Valenti O, Hartwich K, Borhegyi Z, Somogyi P, Klausberger T (2012) Behavior-dependent specialization of identified hippocampal interneurons. Nat Neurosci 15: 1265-1271.

Nabbout R, Chemaly N, Chipaux M, Barcia G, Bouis C, Dubouch C, Leunen D, Jambaqué I, Dulac O, Dellatolas G, Chiron C (2013) Encephalopathy in children with Dravet syndrome is not a pure consequence of epilepsy. Orphanet J Rare Dis 8:176.

Nakashiba T, Buhl DL, McHugh TJ, Tonegawa S (2009) Hippocampal CA3 output is crucial for ripple-associated reactivation and consolidation of memory. Neuron 62:781-787.

National Research Council (2011) Guide for the Care and Use of Laboratory 
Animals, Eighth Edition. Washington, DC: The National Academies Press.

Nguyen DP, Kloosterman F, Barbieri R, Brown EN, Wilson MA (2009) Characterizing the frequency structure of fast oscillations in the rodent hippocampus. Front Integr Neurosci 3:11.

Ogiwara I, Miyamoto H, Morita N, Atapour N, Mazaki E, Inoue I, Takeuchi T, Itohara S, Yanagawa Y, Obata K, Furuichi T, Hensch TK, Yamakawa K (2007) Nav1.1 localizes to axons of parvalbumin-positive inhibitory interneurons: a circuit basis for epileptic seizures in mice carrying an Scn 1a gene mutation. J Neurosci 27:5903-5914.

Ogiwara I, Iwasato T, Miyamoto H, Iwata R, Yamagata T, Mazaki E, Yanagawa Y, Tamamaki N, Hensch TK, Itohara S, Yamakawa K (2013) Nav1.1 haploinsufficiency in excitatory neurons ameliorates seizureassociated sudden death in a mouse model of Dravet syndrome. Hum Mol Genet 22:4784-4804.

Ponomarenko AA, Korotkova TM, Sergeeva OA, Haas HL (2004) Multiple GABAA receptor subtypes regulate hippocampal ripple oscillations. Eur J Neurosci 20:2141-2148.

Ponomarenko AA, Li JS, Korotkova TM, Huston JP, Haas HL (2008) Frequency of network synchronization in the hippocampus marks learning. Eur J Neurosci 27:3035-3042.

Rácz A, Ponomarenko AA, Fuchs EC, Monyer H (2009) Augmented hippocampal ripple oscillations in mice with reduced fast excitation onto parvalbumin-positive cells. J Neurosci 29:2563-2568.

Ragona F, Granata T, Dalla Bernardina B, Offredi F, Darra F, Battaglia D, Morbi M, Brazzo D, Cappelletti S, Chieffo D, De Giorgi I, Fontana E, Freri E, Marini C, Toraldo A, Specchio N, Veggiotti P, Vigevano F, Guerrini R, Guzzetta F, et al (2011) Cognitive development in Dravet syndrome: a retrospective, multicenter study of 26 patients. Epilepsia 52:386-392.

Rubinstein M, Han S, Tai C, Westenbroek RE, Hunker A, Scheuer T, Catterall WA (2015) Dissecting the phenotypes of Dravet syndrome by gene deletion. Brain 138:2219-2233.

Schlingloff D, Káli S, Freund TF, Hájos N, Gulyás AI (2014) Mechanisms of sharp wave initiation and ripple generation. J Neurosci 34:11385-11398.

Shmuely S, Sisodiya SM, Gunning WB, Sander JW, Thijs RD (2016) Mortality in Dravet syndrome: a review. Epilepsy Behav 64:69-74.

Skluzacek JV, Watts KP, Parsy O, Wical B, Camfield P (2011) Dravet syndrome and parent associations: the IDEA league experience with comorbid conditions, mortality, management, adaptation, and grief. Epilepsia 52:95-101.

Stark E, Eichler R, Roux L, Fujisawa S, Rotstein HG, Buzsáki G (2013) Inhibition-induced theta resonance in cortical circuits. Neuron 80: 1263-1276.

Stark E, Roux L, Eichler R, Senzai Y, Royer S, Buzsáki G (2014) Pyramidal cell-interneuron interactions underlie hippocampal ripple oscillations. Neuron 83:467-480.

Suh J, Foster DJ, Davoudi H, Wilson MA, Tonegawa S (2013) Impaired hippocampal ripple-associated replay in a mouse model of schizophrenia. Neuron 80:484-493.

Sullivan D, Csicsvari J, Mizuseki K, Montgomery S, Diba K, Buzsáki G (2011) Relationships between hippocampal sharp waves, ripples, and fast gamma oscillation: influence of dentate and entorhinal cortical activity. J Neurosci 31:8605-8616.

Tai C, Abe Y, Westenbroek RE, Scheuer T, Catterall WA (2014) Impaired excitability of somatostatin- and parvalbumin-expressing cortical interneurons in a mouse model of Dravet syndrome. Proc Natl Acad Sci U S A 111:E3139-E3148.

Takács VT, Cserép C, Schlingloff D, Pósfai B, Szőnyi A, Sos KE, Környei Z, Dénes Á, Gulyás AI, Freund TF, Nyiri G (2018) Co-transmission of acetylcholine and GABA regulates hippocampal states. Nat Commun 9:2848.

Tatsukawa T, Ogiwara I, Mazaki E, Shimohata A, Yamakawa K (2018) Impairments in social novelty recognition and spatial memory in mice with conditional deletion of Scn la in parvalbumin-expressing cells. Neurobiol Dis 112:24-34.

Taxidis J, Coombes S, Mason R, Owen MR (2012) Modeling sharp waveripple complexes through a CA3-CA1 network model with chemical synapses. Hippocampus 22:995-1017.

Timofeev I, Grenier F, Steriade M (2004) Contribution of intrinsic neuronal factors in the generation of cortically driven electrographic seizures. J Neurophysiol 92:1133-1143.

Traub RD (2003) Fast oscillations and epilepsy. Epilepsy Curr 3:77-79.

Vacher H, Mohapatra DP, Trimmer JS (2008) Localization and targeting of voltage-dependent ion channels in mammalian central neurons. Physiol Rev 88:1407-1447.

Valero M, Averkin RG, Fernandez-Lamo I, Aguilar J, Lopez-Pigozzi D, Brotons-Mas JR, Cid E, Tamas G, Menendez de la Prida L (2017) Mechanisms for selective single-cell reactivation during offline sharp-wave ripples and their distortion by fast ripples. Neuron 94:1234-1247.e7.

van der Stelt O, Belger A, Lieberman JA (2004) Macroscopic fast neuronal oscillations and synchrony in schizophrenia. Proc Natl Acad Sci U S A 101:17567-17568.

van de Ven GM, Trouche S, McNamara CG, Allen K, Dupret D (2016) Hippocampal offline reactivation consolidates recently formed cell assembly patterns during sharp wave-ripples. Neuron 92:968-974.

Vandecasteele M, Varga V, Berényi A, Papp E, Barthó P, Venance L, Freund TF, Buzsáki G (2014) Optogenetic activation of septal cholinergic neurons suppresses sharp wave ripples and enhances theta oscillations in the hippocampus. Proc Natl Acad Sci U S A 111:13535-13540.

Wang XJ, Buzsáki G (1996) Gamma oscillation by synaptic inhibition in a hippocampal interneuronal network model. J Neurosci 16:6402-6413.

Wiegand JP, Gray DT, Schimanski LA, Lipa P, Barnes CA, Cowen SL (2016) Age is associated with reduced sharp-wave ripple frequency and altered patterns of neuronal variability. J Neurosci 36:5650-5660.

Ylinen A, Bragin A, Nádasdy Z, Jandó G, Szabó I, Sik A, Buzsáki G (1995) Sharp wave-associated high-frequency oscillation $(200 \mathrm{~Hz})$ in the intact hippocampus: network and intracellular mechanisms. J Neurosci 15: $30-46$.

Yu FH, Mantegazza M, Westenbroek RE, Robbins CA, Kalume F, Burton KA, Spain WJ, McKnight GS, Scheuer T, Catterall WA (2006) Reduced sodium current in GABAergic interneurons in a mouse model of severe myoclonic epilepsy in infancy. Nat Neurosci 9:1142-1149. 